\title{
Fatores prognósticos e gravidade de pacientes com Síndrome da Imunodeficiência Adquirida associada com insuficiência respiratória na unidade de terapia intensiva
}

\author{
Prognostic factors and severity of patients with Acquired \\ Immunodeficiency Syndrome associated with respiratory \\ insufficiency in the intensive care unit
}

Resumo Introdução: A Síndrome da Imunodeficiência Adquirida (SIDA) é uma doença crônica, intensamente relacionada à incidência de afecções oportunistas, como desordens pulmonares.

Objetivo: Identificar o perfil clínico-epidemiológico, fatores relacionados à mortalidade e gravidade de pacientes com SIDA internados na Unidade de Terapia Intensiva associados à Insuficiência Respiratória (IRpA).

Métodos: Estudo prospectivo, quantitativo e observacional realizado na UTI, de abril a novembro de 2017. Foram incluídos pacientes com SIDA e alguma complicação respiratória e coletados dados como: idade, sexo, diagnóstico, tempo de diagnóstico, presença de IRpA, dias de internação na UTI e condições de alta.

Resultados: 32 pacientes, com média de 44,8 anos de idade, a principal causa de internação foi a pneumocistose associada ou não à IRpA. A mortalidade foi de $68,75 \%$ durante um intervalo de oito meses, parcialmente explicada pela gravidade deles, com média no Apache II de 23,27. Conclusão: Esse perfil segue as tendências da epidemia no Brasil: homens, adultos jovens com comprometimento respiratório, onde a maioria evoluiu com óbito.

Palavras-chave: Síndrome da Imunodeficiência Adquirida. HIV. Complicações Respiratórias.

AbSTRACT Introduction: Acquired Immunodeficiency Syndrome (AIDS) is a chronic disease, intensely related to the incidence of opportunistic diseases, such as pulmonary disorders.

Objective: To identify the clinical-epidemiological profile, factors related to mortality and severity of AIDS patients admitted to the Intensive Care Unit associated with Respiratory Insufficiency (PIR).

Francisco Maurílio da Silva CARRIAS $^{1}$

Brena Costa de Oliveira ${ }^{\mathrm{I}}$ Angelo Eduardo Vasconcelos GUIMARÃES ${ }^{\text {II }}$

Matheus da Silveira ArRais ${ }^{I I}$ Gisella Maria Lustoza Serafim ${ }^{\mathrm{II}}$ ${ }^{\mathrm{I}}$ Universidade Federal do Piauí (UFPI), Teresina/PI - Brasil.

II Universidade Estadual do Piauí (UESPI), Teresina/PI - Brasil.
Methods: Prospective, quantitative and observational study carried out in the ICU, in April 2017. Patients with AIDS and some respiratory complications were included and data were collected, such as: age, sex, diagnosis, time of diagnosis, presence of ARF, days of hospitalization in ICU and discharge conditions.

Results: 32 patients, with an average of 44.8 years, the main cause of hospitalization was pneumocystosis associated or not with IRpA. Mortality was $68.75 \%$ during the 8 -month interval, interrupted explained by 
their severity, with an Apache II average of 23.27. Conclusion: This profile follows the trends of the epidemic in Brazil: men, young adults with respiratory impairment, where the majority evolved with death.

Keywords: ACQuired Immunodeficiency Syndrome. HIV. RespiRATORY COMPLICATIONS.

\section{INTRODUÇÃO}

A Síndrome da Imunodeficiência Adquirida (SIDA) é uma doença grave, emergente, que vem se disseminando desde a descrição dos seus primeiros casos em junho de 1981. Globalmente, 36,7 milhões de pessoas em todo o mundo viviam com o vírus da imunodeficiência humana (HIV) em 2016. Um aumento em relação aos anos anteriores, à medida que mais pessoas estão recebendo a terapia antirretroviral que salva vidas e queda progressiva da mortalidade. ${ }^{1}$

O desenvolvimento de novas tecnologias no tratamento com antirretrovirais promoveu o aumento dessa sobrevida, assim como a diminuição na incidência de infecções oportunistas e, por conseguinte, diminuição nas internações hospitalares relacionadas à SIDA. ${ }^{2}$

Desde o início da epidemia, 1980 até 2016, foram registrados no Brasil 35 milhões de óbitos relacionados a essa síndrome. A maior concentração dos casos está nos indivíduos com idade entre 25 e 39 anos para ambos os sexos; entre os homens, essa faixa etária corresponde a 53,6\%, e entre as mulheres $49,8 \%$ do total dos casos. Desde o início da epidemia de SIDA (1980), até dezembro de 2014, foram identificados 290.929 óbitos, tendo como causa básica a SIDA. ${ }^{3}$

Essa mortalidade associada à SIDA está intensamente relacionada à incidência de afecções oportunistas, e muitas dessas afecções apresentam-se como desordens pulmonares, que em $65 \%$ dos casos serão a pri- meira manifestação da doença, e em mais de $80 \%$ dos casos ocorrerão no curso da enfermidade. ${ }^{4}$ Entre as complicações pulmonares associadas predominam as infecciosas, sendo a pneumonia bacteriana, pnemocistose e tuberculose as principais causas de infecções pulmonares e a pneumocistose e tuberculose as principais causas de morbimortalidade. $^{5}$

Pesquisas apontam que cerca de $4 \%$ a $12 \%$ dos pacientes que sobrevivem com SIDA necessitam de internação na unidade de terapia intensiva (UTI). Nos anos 80 , os pacientes admitidos em UTI apresentavam alta mortalidade. Isso, associado aos altos custos das UTIs, alimentou a ideia da futilidade de cuidados intensivos com esses pacientes. ${ }^{6}$

Apesar do acentuado avanço no tratamento da SIDA, ela permanece como um dos mais importantes agravos infecciosos, atualmente. O uso de sistemas de pontuação fisiológica para identificação de pacientes quanto à gravidade de seu estado e fazer previsões de mortalidade ancoradas nessa gravidade tem sido, especialmente, considerado nos últimos anos. Um dos primeiros sistemas de pontuação fisiológica é Acute Physiology and Chronic Health Evaluation II (APACHE II), introduzida por Knaus e colaboradores, em 1985. Esse modelo é calculado com base em 12 critérios fisiológicos, idade e condição prévia do paciente, havendo uma estreita relação entre essa pontuação e a mortalidade hospitalar de pacientes criticamente doentes.? 
Por ser uma síndrome com grandes repercussões sociais, envolvendo aspectos culturais, educacionais e econômicos, em constante crescimento do número de portadores, há uma necessidade de estimar o risco de morte nesses indivíduos. E uma das propostas deste trabalho é a identificação de fatores associados para classificação de grupos vulneráveis, possibilitando o desenvolvimento de estratégias para a prevenção, motivação à adesão à terapêutica e modulação dos esforços de redução de risco, com o objetivo de reduzir a sua mortalidade e o consequente índice de morbimortalidade. Acrescido a isso, soma-se o mérito de fornecer subsídios às autoridades governamentais para o estabelecimento de políticas públicas efetivas. ${ }^{2,7,8}$

Este estudo objetiva identificar o perfil clínico-epidemiológico, os fatores relacionados à mortalidade e gravidade de pacientes com HIV/SIDA internados na UTI associado à insuficiência respiratória.

\section{Métodos}

Desenho do estudo: estudo prospectivo, quantitativo e de caráter observacional.

Local, período da coleta de dados e amostra: foi realizado na Unidade de Terapia Intensiva (UTI) de um hospital público em Teresina - PI, referência no atendimento de doenças tropicais no período de abril a novembro de 2017. A amostra do estudo foi referente aos pacientes com infecção confirmada pelo vírus HIV e que apresentassem a SIDA.

Critérios de inclusão: foram incluídos pacientes admitidos na Unidade de Terapia Intensiva no período da coleta de dados, que tenham tido como causa admissional sintomas respiratórios, que estivessem em sua primeira admissão na UTI, com idade igual ou superior a 18 anos e de ambos os gêneros.

Depois de realizado o recrutamento dos pacientes a fim de encontrar aqueles que se enquadrassem no perfil da população a ser estudada, foi feita uma apresentação dos objetivos dessa pesquisa e do Termo de Consentimento Livre e Esclarecido (TCLE) ao paciente e/ou responsável legal com informações sobre os aspectos éticos e procedimentos da pesquisa. E somente após a aquiescência por meio deste o paciente foi incluso ao estudo.

Coleta de dados: os dados foram coletados de forma manual, diariamente, por meio de fichas padronizadas para esse estudo. Os dados da admissão de cada paciente continham as seguintes informações: identificação, idade, sexo, tempo do diagnóstico, condições clínicas atuais, se fazia uso de ventilação mecânica, uso de drogas vasoativas, se realizava hemodiálise durante internação, presença de leucocitose, choque séptico e uso de antibioticoterapia.

O escore APACHE II foi utilizado como índice de prognóstico nas primeiras 24h, após admissão na UTI, aplicado para avaliação da gravidade clínica e pontuação de mortalidade estimada. Para cada paciente foi calculado o valor dos escores, como também a mortalidade prevista. Os cálculos das mortalidades previstas foram realizados utilizando-se as fórmulas e métodos já estabelecidos para o escore usado. O paciente foi acompanhado até seu desfecho (óbito ou alta) durante sua internação na UTI.

Procedimentos éticos: $\mathrm{O}$ estudo foi pautado nos princípios éticos e legais que envol- 
vem pesquisa com seres humanos, e seguiu as normas da resolução 422/12 do Conselho Nacional de Saúde (CNS), foi aprovado pela Comissão Científica do Hospital e pelo Comitê de Ética e Pesquisa da Universidade Estadual do Piauí (via Plataforma Brasil), conforme número do protocolo $\mathrm{n}^{\circ}$. 1.502.406. Os responsáveis pelos participantes do estudo assinaram o Termo de Consentimento Livre e Esclarecido (TCLE) e receberam informações sobre os pontos principais do estudo.

Organização e Análise dos Dados: Os dados coletados foram submetidos à análise estatística. As variáveis categóricas foram descritas por meio de frequências e porcentagem e as numéricas por intermédio de média \pm desvio padrão. Para comparações de dados categóricos, foram utilizados o teste exato de Fisher e para dados contínuos foram usados o teste não-paramétrico de $T$ Student (para amostras independentes) e para análise do tempo de sobrevivência foi empregado o gráfico de Kaplan Meier. Foi considerado estatisticamente significativo valor de $p<0,05$. As análises estatísticas foram feitas utilizando o programa BioEstat 5.0.

\section{RESUltados}

Durante o período da coleta foram admitidos 118 pacientes na UTI estudada, destes 70 foram internados com o diagnóstico de SIDA. A partir de então foram recrutados os pacientes que tiveram como causa admissional insuficiência respiratória, no total de 38. Foram excluídos cinco pacientes por falta de informações no prontuário e um por ser readmissão, conforme Figura 1.

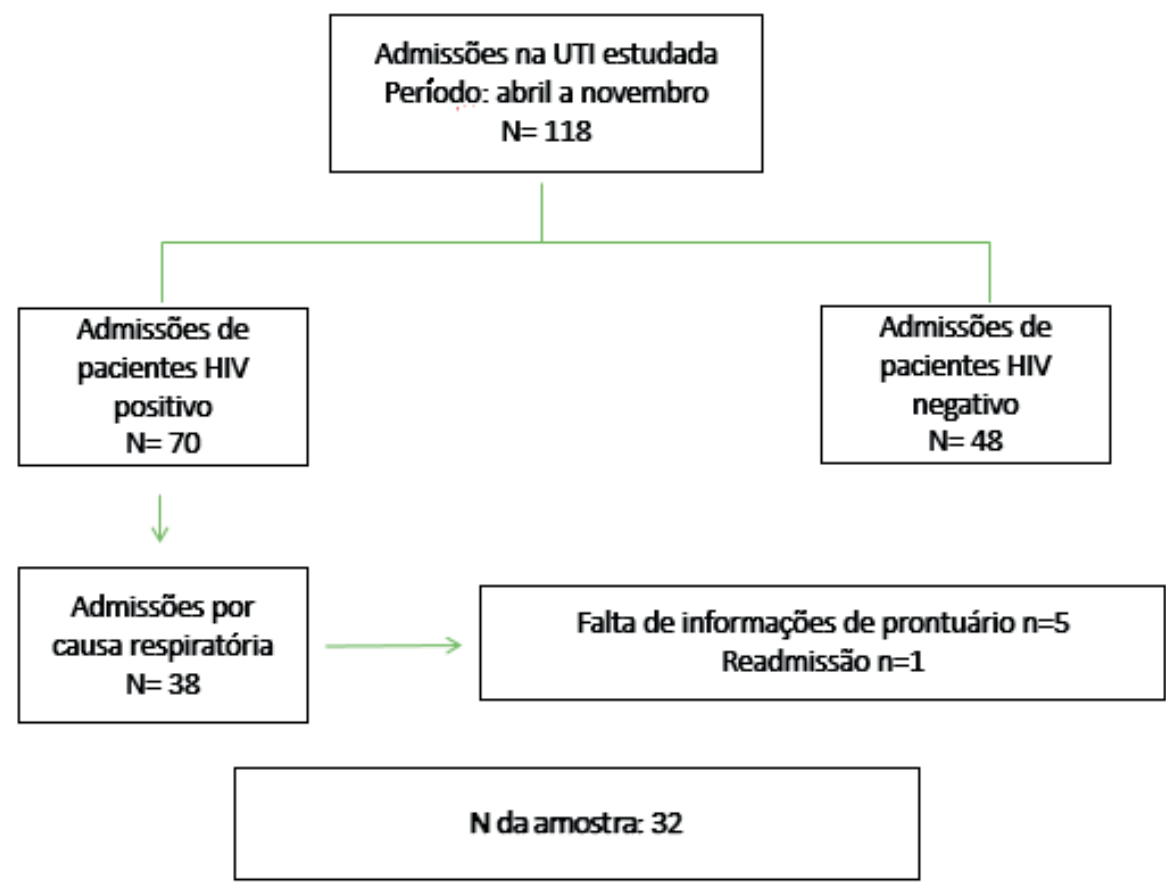

Figura 1: Organograma do estudo 
Foram então avaliados 32 pacientes com SIDA e complicação respiratória durante sua internação na UTI. Quanto ao sexo, havia 24 homens e 8 mulheres, com idade média de $44,8 \pm 13,79$ anos, sendo o paciente mais novo com 22 anos e o mais velho, 68 anos de idade, vide Tabela 1.

Tabela 1: Características clínicas da amostra estudada $(n=32)$.

\begin{tabular}{lcc}
\hline \multicolumn{1}{c}{ Variável } & N & \% \\
\hline $\begin{array}{l}\text { Sexo } \\
\quad \text { Masculino }\end{array}$ & 24 & 75,00 \\
$\quad$ Feminino & 8 & 25,00 \\
\hline $\begin{array}{l}\text { Diagnóstico } \\
\text { Sabidamente } \\
\text { portador }\end{array}$ & 13 & \\
$\begin{array}{l}\text { Recém- } \\
\text { diagnosticado }\end{array}$ & 19 & 59,37 \\
\hline Idade (anos) & \multicolumn{2}{c}{$44,84 / 13,79^{*}$} \\
\hline $\begin{array}{l}\text { Internação na } \\
\text { UTI (dias) }\end{array}$ & \multicolumn{2}{|c}{$15,40 / 10,09^{*}$} \\
\hline
\end{tabular}

Legenda: Dados apresentados com valores médios e desvio padrão.

Fonte: Arquivo dos pesquisadores, Teresina-PI, 2018.
O Gráfico 1 mostra principais complicações que levaram à internação do paciente na UTI.

Observou-se que a necessidade de ventilação mecânica ou aminas vasoativas em qualquer momento na UTI, a necessidade de ventilação mecânica nas primeiras 24 horas da UTI, o choque séptico e o uso de antibioticoterapia, todos foram fatores associados a óbito na UTI, porém apenas o uso de aminas vasoativas com estatística significante, de acordo com $\mathrm{Ta}$ bela 2 e 3 .

Ademais, após o teste de log rank, por meio do gráfico de Kaplan Meier (Gráfico 2), foi possível observar que não houve significância no tempo de sobrevida dos homens e mulheres conforme o tempo de internação, no entanto houve proporcionalidade entre os riscos, visto que os gráficos não se cruzam.

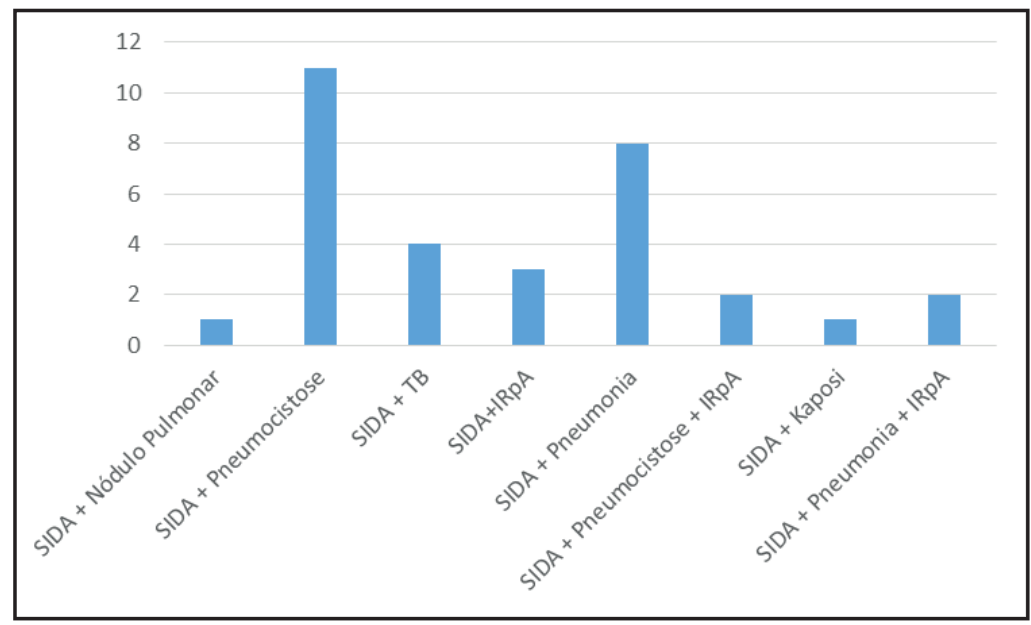

Gráfico 1: Principais diagnósticos encontrados nos pacientes

HIV positivos internados na UTI $(\mathrm{n}=32)$.

Legenda: SIDA - Síndrome da Imunodeficiência Adiquirida, TB

- Tuberculose e IRpA - Insuficiência Respiratória Aguda.

Fonte: Arquivo dos pesquisadores, Teresina-PI, 2018. 
Tabela 2: Análise comparativa entre os pacientes que evoluíram com óbito e alta hospitalar, segundo as variáveis analisadas pelo teste exato de fisher $(n=32)$. Teresina-PI, 2018.

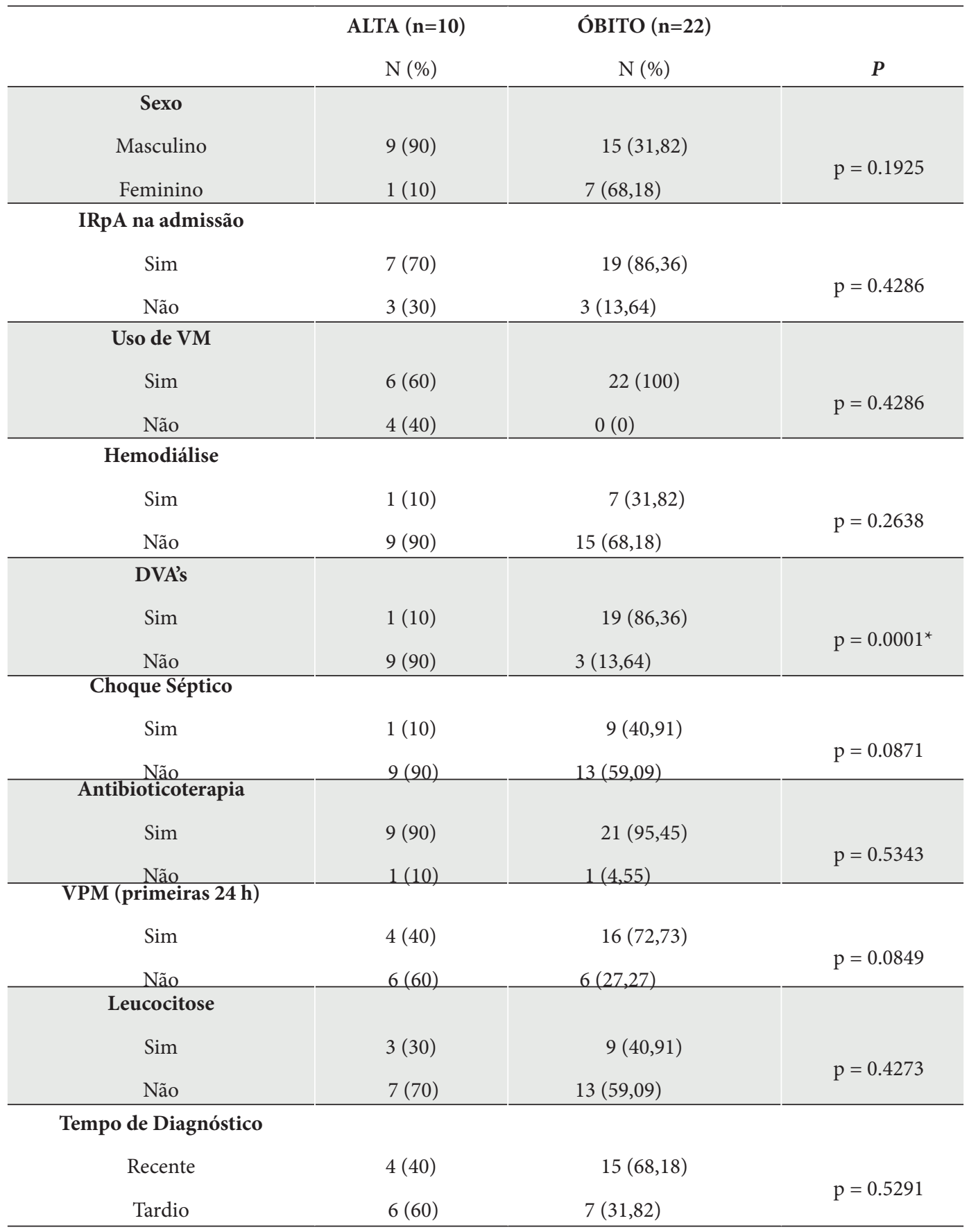


Tabela 3: Análise comparativa entre os pacientes que evoluíram com óbito e com alta hospitalar, segundo as variáveis analisadas pelo teste t student $(n=32)$. Teresina-PI, 2018.

\begin{tabular}{cccc}
\hline & $\begin{array}{c}\text { ALTA }(\mathrm{n}=10) \\
\text { Média/DP }\end{array}$ & $\begin{array}{c}\text { ÓBITO }(\mathrm{n}=22) \\
\text { Média/DP }\end{array}$ & $P$ \\
\hline Idade (anos) & $49.0+10.44$ & $42.5+14.90$ & 0,1285 \\
\hline Tempo UTI (dias) & $15.1+5.98$ & $15.54+11.62$ & 0,4437 \\
\hline APACHE & $16.1+6.13$ & $23.27+7.07$ & $0,0048^{*}$ \\
\hline CATEGORIA APACHE (\%) & $23.80+14.14$ & $46.36+22.63$ & $0,0035^{*}$ \\
\hline
\end{tabular}

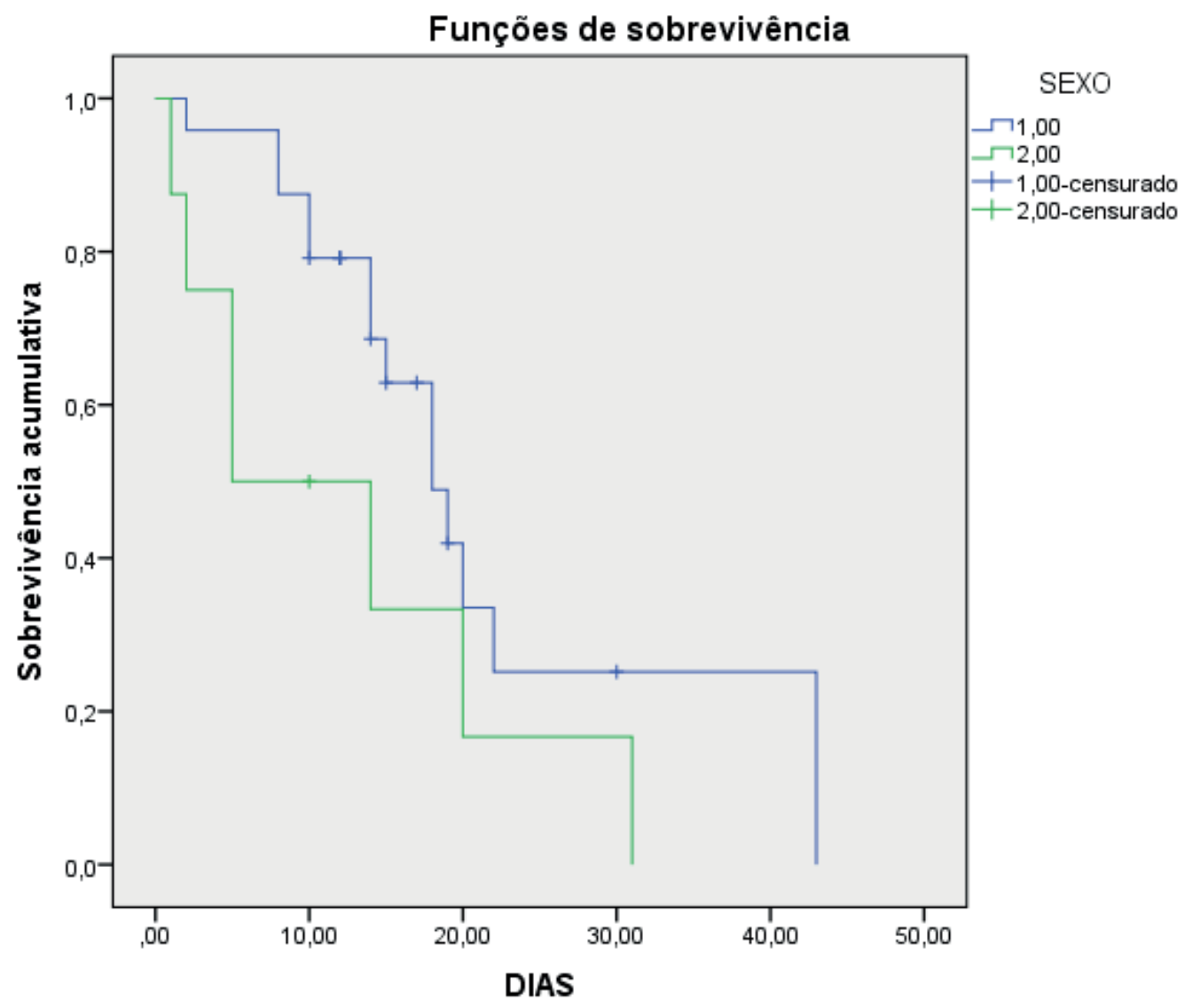

Gráfico 2: Teste de igualdade de distribuições de sobrevivência para os diferentes sexos.

Comparações globais

\begin{tabular}{|l|r|r|r|}
\hline & Qui-quadrado & df & Significância. \\
\hline Log Rank (Mantel-Cox) & 2,876 & & 1 \\
\hline
\end{tabular}

Legenda: 1(azul): homens; 2(verde): mulheres

Fonte: Arquivo dos pesquisadores, Teresina-PI, 2018. 


\section{Discussão}

No presente estudo, $75 \%$ dos pacientes que internaram na UTI com diagnóstico de SIDA, e com admissão associada a causas respiratórias, eram do sexo masculino, e $25 \%$ do sexo feminino. Esses dados refletem a realidade dos pacientes com essa doença infecciosa, onde as taxas de detecção de SIDA em homens nos últimos dez anos têm apresentado tendência de crescimento no Brasil. Em 2005, a taxa foi de 24,7 casos para cada 100 mil habitantes, que passou para 27,7 em 2014, representando um aumento de $10,8 \%$. Entre as mulheres, observa-se tendência de queda dessa taxa nos últimos dez anos, a qual passou de 16,3 casos a cada 100 mil habitantes em 2005 e para 13,7, em 2014, representando uma queda de $18,9 \%{ }^{3}$

Em um estudo epidemiológico de uma UTI adulto localizado em Juiz de Fora se constatou que $52,6 \%$ dos pacientes internados eram do sexo masculino e $47,4 \%$ do sexo feminino. ${ }^{8}$ Com relação à idade, a média foi de $48,29 \pm 13$ anos, o que é compatível com alguns estudos brasileiros que apontam que a média de idade varia de 36,5 a 50 anos. ${ }^{9}$

Apesar dos métodos de diagnóstico do HIV estarem disponíveis e serem gratuitos pelo sistema público de saúde, nesse estudo observou-se que $59,37 \%$ dos pacientes apresentaram diagnóstico do HIV há menos de um mês. Esses dados indicam que parcela significativa dos pacientes exibiu diagnóstico recente ou início tardio da terapia antirretroviral (TARV), o que sugere falhas no diagnóstico e no início do tratamento do paciente HIV positivo.

O diagnóstico tardio não é um problema sugerido apenas neste estudo. Um estudo realizado em 31 países da União Europeia e do Espaço Econômico Europeu destaca que uma proporção significativa de indivíduos não pode tirar o máximo proveito do tratamento da infecção do HIV por diagnóstico tardio da doença. ${ }^{10} \mathrm{O}$ diagnóstico tardio está associado a uma mortalidade quase 10 vezes maior, no ano seguinte ao diagnóstico, a uma maior morbidade e transmissão do HIV antes de ser diagnosticado e tratado, além de um grande risco de eventos clínicos e hospitalizações, onerando os sistemas de saúde. ${ }^{11,12}$

Nesse estudo, a principal causa que levou o paciente a ser internado na UTI, foi a pneumocistose. Dos pacientes, $31,25 \%$ tinham como diagnóstico Pneumonia, 12,25\% Tuberculose Pulmonar, 3,1\% diagnóstico de Kaposi pulmonar, e 3,1\% Nódulo Pulmonar. Em um estudo realizado em Paris nos anos de 1996-2005, 18,7\% dos pacientes apresentaram a Pneumocistose como diagnóstico no momento da internação. ${ }^{13}$ Vale ressaltar que o diagnóstico de pneumocistose no hospital estudado é dado pela clínica do paciente associado a exames de imagem e laboratoriais, pois o mesmo não apresenta o lavado brônquico ou biópsia do parênquima pulmonar.

Na França, 38,8\% dos pacientes internados tinham como causa a infecção respiratória aguda, ${ }^{14}$ similar a um estudo realizado em São Paulo, em que 46\% dos pacientes foram internados na UTI com diagnóstico de infecção respiratória aguda, ${ }^{15}$ o que de fato confirma que as doenças pulmonares são as principais causas de morbidade e mortalidade entre os pacientes infectados pelo HIV. ${ }^{16}$

A mortalidade dos pacientes HIV positivos admitidos na UTI estudada foi de $68,75 \%$ durante o intervalo de oito meses. 
Essa alta mortalidade pode ser parcialmente explicada pela gravidade dos pacientes na ocasião da admissão na UTI, com média no Apache II de 23,27, uma pontuação muita alta de acordo com a literatura. Além disso, a maioria dos pacientes foi admitida por causas relacionadas à AIDS, com frequente necessidade de ventilação mecânica e elevado número de admissões por sepse grave, duas condições sabidamente associadas ao pior prognóstico. Ademais, os escores preditores de mortalidade apresentaram médias mais altas que as encontradas por outros autores, o que sugere maior gravidade dos pacientes à admissão. Um estudo realizado em uma UTI em Uganda, África, mostrou o índice médio do APACHE II de 24 e revelou que $50,88 \%$ dos pacientes que evoluíram a óbito eram do sexo feminino. ${ }^{17}$

Quanto maior o índice do APACHE II tanto maior é a probabilidade estimada para a mortalidade do paciente. Um estudo realizado com 73 pacientes infectados pelo HIV internados na UTI do Hospital das Doenças Infecciosas de São José, Fortaleza, Brasil evidenciou que os pacientes com maiores índices do APACHE II foram a óbito, média de $65,1 \%$ (óbito) contra $42,5 \%$ (alta). Contudo, um estudo retrospectivo, caso-controle, realizado na UTI no Hospital St. Barnabas, de Nova York, destaca que o escore APACHE II médio foi de 18 entres os pacientes HIV-positivos e HIV-negativos. ${ }^{18}$

Outro dado relevante foi que todos os pacientes desse estudo que apresentaram infecção respiratória aguda na internação e uso de ventilação mecânica foram a óbito, fato justificado pela literatura, que enfatiza que a falha de órgãos é manifestada pela necessidade da ventilação mecânica e esses possuem 4,5 vezes mais chances de morrer. ${ }^{17,19}$ Além disso, corroborando com esses dados, um estudo realizado em pacientes com infecção respiratória, infectados pelo HIV internados na UTI, destacou que $92,7 \%$ dos pacientes que foram a óbito utilizaram ventilação mecânica ${ }^{18}$ e $\operatorname{Croda}^{20}$ observou que a ventilação mecânica nas primeiras 24 horas de UTI também permaneceu associada ao óbito na UTI.

As características dos pacientes que sobreviveram à internação na UTI foram comparadas com as daqueles que evoluíram para óbito na UTI, nesse estudo. Houve diferença significativa $(p<0,05)$ entre sobreviventes e não-sobreviventes quanto ao uso de drogas vasoativas. A média do escore APACHE II também foi significativamente diferente entre os grupos de sobreviventes e não-sobreviventes $(0,0048)$. Ratificando os dados já apresentados, a mortalidade hospitalar foi ligada ao uso prolongado de drogas vasoativas em um estudo feito na capital do Chile. ${ }^{21}$ Esse fator tem sido identificado com o aumento da mortalidade em estudos como o realizado em Brasília. ${ }^{2}$

Altos escores do APACHE II, ventilação mecânica, tratamento da tuberculose, terapia antirretroviral e choque séptico são descritos como fatores associados a um maior índice de mortalidade na UTI, ${ }^{2}$ assim como, sepse mais grave, com níveis mais altos de marcadores inflamatórios do que os pacientes HIV- negativos. ${ }^{22}$ Pacientes com HIV positivo, internados na UTI do Hospital Militar de Santiago, que apresentaram idade maior que 50 anos e APACHE II maior ou igual a 18 pontos foram significativamente associados com uma menor sobrevida a um ano e meio de pós-alta. ${ }^{21}$ 
O conhecimento do perfil dessa população, assim como, a utilização de um preditor de mortalidade, amplamente usado e eficaz, corroboram para intervir de maneira sistematizada na assistência prestada a esse paciente. Por conseguinte, melhoram os desfechos nas UTI e na qualidade de vida desse paciente, fato que demonstra a importância desse estudo, bem como, sua grande relevância clínica, pois seus achados se ratificam na literatura e incrementam as pesquisas, nessa área, realizadas no Nordeste. Além disso, vale destacar que a pesquisa possui limitações que estão relacionadas ao fato de avaliar um ambiente único, bem como, sua pequena amostra estudada, sendo necessários maiores estudos sobre o tema em questão.

\section{CONSIDERAÇões FINAIS}

Por meio desse estudo, nota-se que o perfil clínico-epidemiológico dos pacientes com SIDA, que participaram dessa pesquisa, segue as tendências da epidemia no Brasil: homens, adultos jovens com comprometimento respiratório, em que a maioria evoluiu com óbito. Além disso, percebe-se que existem alguns fatores relacionados ao maior índice de mortalidade e gravidade desses pacientes, como sepse grave, insuficiência respiratória à admissão, necessidade de ventilação mecânica ou de aminas vasoativas e uso de antibioticoterapia, embora apenas o uso de aminas vasoativas tenha sido estatisticamente significativo.

\section{REFERÊNCIAS}

1. UNAIDS. Global report: UNAIDS report on the global AIDS epidemic 2017. Geneva: UNAIDS, 2017.

2. Amâncio FF. Mortalidade e fatores prognósticos em pacientes HIV positivo internados em unidade de terapia intensiva de hospital especializado em doenças infecto-parasitárias [dissertação]. Belo Horizonte: Faculdade de Medicina da Universidade Federal de Minas Gerais, 2010.

3. Brasil. Ministério Da Saúde. Secretaria de Vigilância em Saúde - Departamento de DST, Aids e Hepatites Virais. Boletim Epidemiológico - Aids e DST, 2015.

4. Chiang $\mathrm{HH}$ et al. Admissions to intensive care unit of HIV-infected patients in the era of highly active antiretroviral therapy: etiology and prognostic factors. Crit Care. 2011, 15 (4): 202.

5. Girardi E., Sabin CA, Monforte AD. Late diagnosis of HIV infection: epidemiological features, consequences and strategies. J Acquir Imunne Defic Syndr. 2007, 46 (Suppl 1).

6. Dickson SJ et al. Survival of HIV-infected patients in the intensive care unit in the era of highly active antiretroviral therapy. Thorax. 2007, 62 (11): 964-96.

7. Giamarellos-Bourboulis EJ et al. Risk assessment in sepsis: a new prognostication rule by APACHE II score and serum soluble urokinase plasminogen activator receptor. Critical care. 2012, 16 (4): 149.

8. Almeida MC; Almeida EB. Perfil dos pacientes com infecção por HIV admitidos em unidade de terapia intensiva adulto em hospital universitário de Juiz de Fora, MG. Rev. de Enferm. da UFJF. 2015, 1 (2): 187-193.

9. Carvalho FL et al. Perfil epidemiológico dos indivíduos HIV positivo e coinfecção HIV-Leishmania em um serviço de referência em São Luís, MA, Brasil. Ciênc. saúde coletiva [online]. 2013, 18 (5): 1.305-1.312. 
10. Pharris A. et al. Ten years after Dublin: principal trends in HIV surveillance in the EU/EEA, 2004 to 2013. Euro Surveill. 2014, 19 (47): 20.968.

11. Ingle $S M$ et al. Impact of risk factors for specific causes of death in the first and subsequent years of antiretroviral therapy among HIV-infected patients. Clin Infect Dis. 2014, 59 (2): 287-97.

12. Oliva J. et al. Predictors of advanced disease and late presentation in new HIV diagnoses reported to the surveillance system in Spain. Gac Sanit. 2014, 28 (2): 116-22.

13. Coquet C. et al. Survival trends in critically ill HIV-infected patients in the highly active antiretroviral therapy era. Crit Care. 2010,14 (3): 107.

14. Morquin D. et al. Short-and long-term outcomes of HIV-infected patients admitted to the intensive care unit: impact of antiretroviral therapy and immunovirological status. Annals of intensive care. 2012, 2 (1): 25.

15. Souza PN. Cuidados paliativos no paciente com HIV/AIDS internado na unidade de terapia intensiva. Rev. bras. ter. intensiva [Internet]. 2016, 28 (3): 301-9.

16. Morris A. et al. American Thoracic Society Committee on HIV Pulmonary Disease. An Official ATS Workshop Report: Emerging Issues and Current Controversies in HIV-Associated Pulmonary Diseases. Proc Am Thorac Soc. 2011, 8 (1): 17-26.

17. Kwizera A. et al. Clinical Characteristics and Short-Term Outcomes of HIV Patients Admitted to an African Intensive Care Unit. Critical care research and practice. 2016: 2610873.

18. Luna LDS et al. Clinical Characteristics, Outcomes and Risk Factors for Death Among Critically Ill Patients With Hiv-Related Acute Kidney Injury. Rev. Inst. Med. trop. S. Paulo. 2016, 58 (52).

19. Meybeck A. et al. Should highly active antiretroviral therapy be prescribed in critically ill HIVinfected patients during the ICU stay? A retrospective cohort study. AIDS Research and Therapy. 2012, 9 (1): 1-9.

20. Croda J. et al. Benefit of antiretroviral therapy on survival of human immunodeficiency virusinfected patients admitted to an intensive care unit. Crit Care Med. 2009, 37 (5): 1.605-1.611.

21. Verdugo F. et al. Pacientes con infección por VIH/SIDA en una Unidad de Pacientes Críticos: La experiencia de un hospital general en un país en desarrollo. Rev. chil. infectol. 2015, 32 (3): 294-303.

22. Silva JM Jr; Santos S. de S. Sepsis in AIDS patients: clinical, etiological and inflammatory characteristics. J Int AIDS Soc. 2013: 16 (1): 17.344.

\section{DAdos DOS AUTORES}

\section{Francisco Maurilio da Silva Carrias}

Pós graduado em Terapia Intensiva pela Universidade Estadual do Piauí (UESPI). Atualmente trabalha no Instituto de doenças tropicais Natan Portela. Teresina/PI - Brasil. mauriliocarrias@gmail.com

\section{Brena Costa de Oliveira}

Fisioterapeuta Residente do HU- Universidade Federal do Piauí (UFPI). Teresina/PI - Brasil. brena_oliveira.5@hotmail.com

\section{ÂNgelo Eduardo Vasconcelos Guimarães}

Pós graduado em Terapia Intensiva pela Universidade Estadual do Piauí (UESPI). Atualmente trabalha no Instituto de Doenças Tropicais Natan Portela. Teresina/PI - Brasil. angelo.fisio91@gmail.com 


\section{Matheus da Silveira Arrais}

Graduando em fisioterapia pela Universidade Estadual do Piauí (UESPI). Teresina PI/Brasil. matheus_arrais7@hotmail.com

\section{Gisela Maria Lustoza Serafim}

Fisioterapeuta do Instituto de Doenças Tropicais Natan Portela. Teresina/PI - Brasil. gisellaserafim@ yahoo.com.br

Submetido em: 17-7-2020

Aceito em: 15-8-2020 